\title{
Effective field theory for long-range properties of bottomonium
}

\author{
Gastão Krein ${ }^{1, a}$ \\ ${ }^{1}$ Instituto de Física Teórica, Universidade Estadual Paulista \\ Rua Dr. Bento Teobaldo Ferraz, 271 - Bloco II, 01140-070 São Paulo, SP, Brazil
}

\begin{abstract}
In this communication we present selected results from a recent study [N. Brambilla, G. Krein, J. Tarrús Castellà and A. Vairo, Phys. Rev. D 93, 054002 (2016)] of long-range properties of bottomonium. An analytical expression for the chromopolarizability of $1 \mathrm{~S}$ bottomonium states is derived within the framework of potential nonrelativistic QCD (pNRQCD). Next, after integrating out the ultrasoft scale associated with the binding energy of bottomonium, the QCD trace anomaly is used to obtain the two-pion production amplitude for the chromopolarizability operator and the result is matched to a chiral effective field theory having bottomonium states and pions as degrees of freedom. We present results for the leading chiral logarithm correction to the mass of the $1 \mathrm{~S}$ bottomonium and the van der Waals potential between two bottomonium states.
\end{abstract}

\section{Introduction}

In this communication we present selected results from a recent study [1] of long-range properties of bottomonium. Our main interest is on the long-range part of the interaction between two heavy quarkonia, where the interaction is the color van der Waals $(\mathrm{vdW})$ force due to the chromopolarizability of the two (color neutral) hadrons, similar to the well-known electric polarizability in atomic physics. The motivation for the study is basically twofold: (1) the structure of some of the new multiquark XYZ hadrons [2,3] might consist of a tightly bound charm quark-antiquark pair interacting with light quarks via color vdW-type of forces [4], (2) quarkonium states can form bound states with atomic nuclei due to color vdW forces [5]. With respect to point (2), a recent lattice QCD calculation [6] found that the binding energy of charmonium in nuclear matter is of the order of $40 \mathrm{MeV}$ or smaller, a result that is in fair agreement with recent phenomenological calculations [7-9]. Given the potential relevance of color van der Waals forces for the understanding of the new XYZ hadrons and possible existence of exotic nuclear bound states, a better understanding of their properties within QCD is required. Although the use of models is vitally important for getting insight and provide guidance to experiments, it is desirable to employ a theoretical framework rooted in QCD, and built on controllable approximations that can be systematically improved. The work in Ref. [1] is a first quantitative attempt in this direction. The framework used is the one of effective field theory (EFT); more specifically, the work is based on nonrelativistic effective field theories [10] and chiral effective field theories $[11,12]$.

ae-mail: gkrein@ift.unesp.br 
The work of Ref. [1] builds on the following line reasoning. The mass of the heavy quark and antiquark $m$ is the largest scale in a quarkonium bound state. Other two relevant scales, given in terms of $m$ and the relative velocity $v$ between the heavy quark and antiquark in the bound state, are the relative momentum $|\mathbf{p}| \sim m v$, and the relative kinetic energy $E \sim m v^{2}$. Another important scale is $\Lambda_{\mathrm{QCD}}$, the scale associated with strong coupling. We assume that the following hierarchy is fulfilled:

$$
m \gg m v \gg m v^{2} \gg \Lambda_{\mathrm{QCD}} .
$$

The assumption can be questioned for some heavy quarkonium states, but once assumed, one can construct in a rigorous way an EFT appropriate to describe the color vdW force. This is achieved through a chain of EFTs by sequentially integrating out degrees of freedom at different scales that appear in the interaction. Integration of the hard scale, $m$, leads to nonrelativistic QCD (NRQCD) [13, 14], appropriate to describe physics at the soft scale $(m v)$. To treat heavy quark-antiquark bound states, it is advantageous to integrate out the soft scales; this gives rise to an EFT named potential nonrelativistic QCD (pNRQC) $[15,16]$ - one advantage is that the EFT contains a single scale, the ultrasoft (US) $\sim m v^{2}$, which makes the power counting simpler; a further advantage is that a pNRQCD calculation of heavy quarkonium bound states resembles a quark model calculation based on the Schrödinger equation. In an interaction between two heavy quarkonia at energies much below than their binding energy $E_{B}$, where $E_{B} \sim m v^{2} \approx m \alpha_{s}^{2} \gg \Lambda_{\mathrm{QCD}}$, the internal structure of the quarkonia is not resolved and one can treat the quarkonium states in terms of independent color-singlet fields. That is, one can integrate out the ultra-soft scale $m \alpha_{s}^{2}$ and match pNRQCD to an EFT [18], that we name gluonic van der Waals EFT (gWEFT), whose degrees of freedom are color-singlet quarkonium and gluon fields at the scale $\Lambda_{\mathrm{QCD}}$, with a coupling given by the polarizability. At the scale of $\Lambda_{\mathrm{QCD}}$, gluons are nonperturbative and their interactions cannot be treated in perturbation theory. However, one can make use of the QCD trace anomaly to match the quarkonium-gluon interaction into a quarkoniumpion interaction, which leads to a new EFT $(\chi$ EFT) with quarkonium and pion degrees of freedom. Finally, to treat quarkonium-quarkonium interactions involving momentum transfers of the order of the pion mass, one can integrate out the pion, since its mass is larger than the relative kinetic energy of the two quarkonia in the interaction. This leads to an EFT (WEFT) that describes the interaction between two quarkonia in terms of an effective potential, the vdW potential. In the next sections we discuss the obtention of the several EFTs, starting from pNRQCD and ending at WEFT-Fig. 1 presents an illustration of the sequence of matching processes involved in the sequence of EFTs: $\mathrm{pNRQCD} \rightarrow \mathrm{gWEFT} \rightarrow \chi \mathrm{EFT} \rightarrow$ WEFT.

The paper is organized as follows. In the next section, pNRQCD is briefly reviewed, we introduce gWEFT and perform the matching by computing the chromopolarizability. In Sec. 3, we use the trace anomaly in QCD to match gWEFT to $\chi$ EFT and obtain the leading chiral logarithm contribution to the heavy quarknium mass. In Sec. 4 we match $\chi$ EFT to WEFT and obtain the van der Waals potential between two quarkonia as the matching coefficient. Selected numerical results are presented in Sec. 5 . Conclusions and Perspectives are presented in Sec. 6.

\section{From pNRQCD to gWEFT: the chromopolarizabilty}

Because of the assumed position of $\Lambda_{\mathrm{QCD}}$ in the hierarchy of scales in Eq. (1), one is in a situation of weak coupling and all the degrees of freedom above $\Lambda_{\mathrm{QCD}}$ can be integrated out analytically using perturbation theory. For the problem of interest here, the Lagrangian density of (weakly coupled) pNRQCD can be written in terms of (color singlet and color octet) fields representing the quarkantiquark pair, US gluons and light quarks - we refer the reader to Ref. [17] for a recent pedagogical review on the technical aspects involved in the derivations. The quark-antiquark fields depend on 
(A) $\mathrm{pNRQCD} \rightarrow \mathrm{gWEFT}$

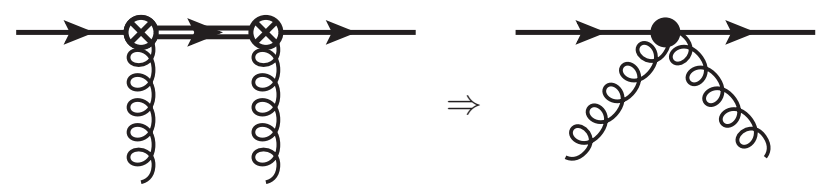

(B) $\mathrm{gWEFT} \rightarrow \chi \mathrm{EFT}$

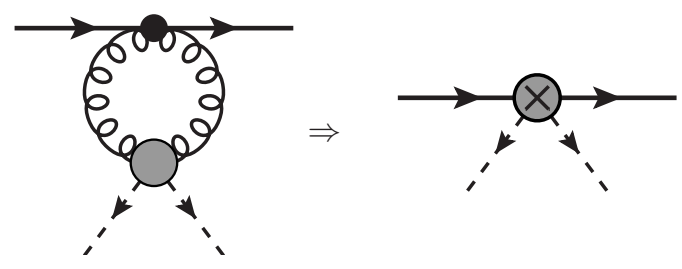

(C) $\chi \mathrm{EFT} \rightarrow$ WEFT
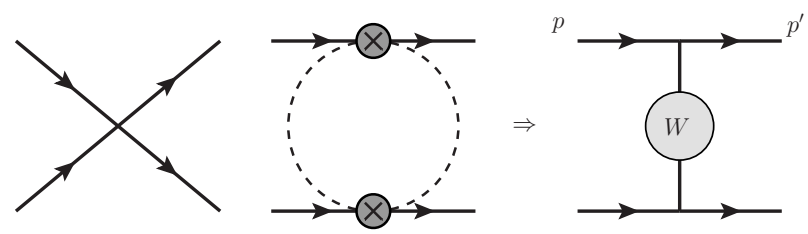

Figure 1. The chain of EFTs constructed to obtain the van der Waals potential for the interaction of two heavy quarkonia. Single solid lines: color singlet quarkonium; Double solid lines: quark-antiquark octet propagators, Wiggled lines: gluons; Dashed lines: pions. (A) pNRQCD $\rightarrow$ gWEFT: circle with the cross represents the chromoelectric dipole vertex of the pNRQCD Lagrangian in Eq. (3). (B) gWEFT $\rightarrow \chi$ EFT: the shaded circle connecting two gluons and two pions represents the matrix element in Eq. (12) from the QCD trace trace anomaly. (C) $\chi \mathrm{EFT} \rightarrow$ WEFT: the blob with $\mathrm{W}$ on the right-hand side graph represents the van der Waals potential. Graphs from Ref. [1].

two coordinates; it is convenient to employ center-of-mass $\boldsymbol{R}$ and relative $\boldsymbol{r}$ coordinates. Since the relative distance between the heavy quark and antiquark in the bound state is of the order of the soft scale, which has been integrated out, the US gauge and light-quark fields depend only on $\boldsymbol{R}$ - this corresponds to a multipole expansion of the US fields. At leading order in $1 / m$ and at $O(r)$ in the multipole expansion $[15,16]$, one has that the action of pNRQCD can be written as

$$
S_{\mathrm{pNRQCD}}=\int d t d \boldsymbol{R} \mathcal{L}_{p \mathrm{NRQCD}}
$$

where the Lagrangian density is given by

$$
\begin{aligned}
\mathcal{L}_{\mathrm{pNRQCD}}= & \mathcal{L}_{\text {light }}+\int d^{3} r\left\{\operatorname{Tr}\left[\mathrm{S}^{\dagger}\left(i \partial_{0}-h_{\mathrm{s}}\right) \mathrm{S}+\mathrm{O}^{\dagger}\left(i D_{0}-h_{\mathrm{o}}\right) \mathrm{O}\right]\right. \\
& \left.+g V_{A}(r) \operatorname{Tr}\left[\mathrm{O}^{\dagger}(\boldsymbol{r} \cdot \boldsymbol{E}) \mathrm{S}+\mathrm{S}^{\dagger}(\boldsymbol{r} \cdot \boldsymbol{E}) \mathrm{O}\right]+\frac{g}{2} V_{B}(r) \operatorname{Tr}\left[\mathrm{O}^{\dagger}(\boldsymbol{r} \cdot \boldsymbol{E}) \mathrm{O}+\mathrm{O}^{\dagger} \mathrm{O}(\boldsymbol{r} \cdot \boldsymbol{E})\right]\right\},
\end{aligned}
$$


where $\mathcal{L}_{\text {light }}$ describes the light degrees of freedom

$$
\mathcal{L}_{\text {light }}=-\frac{1}{2} \operatorname{Tr} \mathrm{G}^{\mu v} \mathrm{G}_{\mu v}+\sum_{\mathrm{i}=1}^{\mathrm{N}_{\mathrm{f}}} \overline{\mathrm{q}}_{\mathrm{i}}\left(\not D-\mathrm{m}_{\mathrm{q}}\right) \mathrm{q},
$$

and $N_{f}$ is the number of flavors, $\mathrm{S}=\mathrm{S}(t, \boldsymbol{R}, \boldsymbol{r})$ and $\mathrm{O}=\mathrm{O}(t, \boldsymbol{R}, \boldsymbol{r})$ are respectively the quarkantiquark matrix-valued singlet and octet fields, normalized with respect to color, $S=1 S / \sqrt{N_{c}}$ and $\mathrm{O}=T^{a} O^{a} / \sqrt{T_{F}}$, with $N_{c}=3$ the number of colors and $T_{F}=1 / 2$, and $h_{\mathrm{s}}$ and $h_{\mathrm{o}}$ are the singlet and octet Hamiltonians

$$
h_{\mathrm{s}}=-\frac{\boldsymbol{\nabla}_{\boldsymbol{r}}^{2}}{m}-\frac{\boldsymbol{\nabla}_{\boldsymbol{R}}^{2}}{4 m}+V_{\mathrm{s}}(r), \quad h_{\mathrm{o}}=-\frac{\boldsymbol{\nabla}_{\boldsymbol{r}}^{2}}{m}-\frac{\boldsymbol{D}_{\boldsymbol{R}}^{2}}{4 m}+V_{\mathrm{o}}(r),
$$

with the potentials given by

$$
V_{\mathrm{s}}(r)=-C_{F} \frac{\alpha_{s}}{r}, \quad V_{\mathrm{o}}(r)=\frac{\alpha_{s}}{2 N_{c} r}, \quad V_{A}(r)=V_{B}(r)=1,
$$

where $C_{F}=\left(N_{c}^{2}-1\right) /\left(2 N_{c}\right)$. Also, $v$ can be identified with $\alpha_{s}$. Finally, as said above, the light-quark and gauge fields are functions of time and $\boldsymbol{R}: q_{i}=q_{i}(t, \boldsymbol{R}), \bar{q}_{i}(t, \boldsymbol{R}), G^{\mu v}=G^{\mu v}(t, \boldsymbol{R}), \boldsymbol{E}^{i} \equiv G^{i 0}(\boldsymbol{R}, t)$, $i D_{0} O \equiv i \partial_{0} O-g\left[A_{0}(\boldsymbol{R}, t), O\right]$.

To be concrete, we concentrate in the $1 S$ color-singlet eigenstates in the bottomonium sector: the $\eta_{b}$ (spin singlet) and $\Upsilon(1 \mathrm{~S})$ (spin triplet). Because of the large bottom mass, spin-dependent interactions are suppressed and we consider these two states as degenerate and describe them by a $0^{-+}$ field $\phi$. In integrating out the scales $m, m \alpha_{s}$ and $m \alpha_{s}^{2}$ from the QCD Lagrangian, one can organize the different terms in the Lagrangian of gWEFT in a power series of the ratios $m \alpha_{s} / m, m \alpha_{s}^{2} /\left(m \alpha_{s}\right)$ and $\Lambda_{\mathrm{QCD}} /\left(m \alpha_{s}^{2}\right)$ as

$$
\mathcal{L}_{\mathrm{gWEFT}}=\mathcal{L}_{\text {light }}+\phi^{\dagger}(t, \boldsymbol{R})\left(i \partial_{0}-E_{\phi}+\frac{\boldsymbol{\nabla}_{\boldsymbol{R}}^{\mathbf{R}}}{4 m}+\frac{1}{2} \beta g^{2} \boldsymbol{E}_{a}^{2}+\cdots\right) \phi(t, \boldsymbol{R}),
$$

where $\cdots$ stands for higher-order operators (like relativistic kinetic corrections or other operators coupling $\phi$ to gluons) and $\beta$, the matching coefficient, is the chromopolarizability, given by [19-22]

$$
\beta=-\frac{2 V_{A}^{2} T_{F}}{3 N_{c}}\left\langle\phi\left|\boldsymbol{r} \frac{1}{E_{\phi}-h_{\mathrm{o}}} \boldsymbol{r}\right| \phi\right\rangle
$$

with $|\phi\rangle$ being a $1 S$ Coulombic state. We note that an important element in Eq. (8) is the characterization of the intermediate states, because of the presence of the octet Hamiltonian $h_{\mathrm{o}}(\boldsymbol{r})$ in the denominator, they must be color-octet states. In weakly coupled pNRQCD, $h_{\mathrm{o}}(\boldsymbol{r})$ is a repulsive Coulomb potential and so the octet intermediate states correspond to Coulombic continuum eigenstates. A distinctive feature of our calculations of the vdW potential Section 4, is the use of polarizability calculated with the exact eigenstates of $h_{\mathrm{o}}(\boldsymbol{r})$, instead of free plane waves commonly used in previous similar calculations. This allows us to assess the large- $N_{c}$ behavior of the polarizability, as the use of plane waves in Eq. (8) corresponds to the $N_{c} \rightarrow \infty$ limit. Explicit numerical results will be presented in Section 5.

\section{From gWEFT to $\chi$ EFT: chiral-log contribution to the quarkonium mass}

At energies of order $m_{\pi} \gg \Lambda_{Q C D}$, the relevant degrees of freedom are the bottomonium states and the (pseudo) Goldstone bosons of dynamical chiral symmetry breaking of QCD, it is natural to integrate out the gluons in favor of pions. However, as already mentioned, the integration cannot be done 
perturbatively as the gluons are strongly coupled at scales of the order of $\Lambda_{\mathrm{QCD}}$ and below. However, from the trace anomaly of QCD, one has a matrix element connecting two-chromoelectric field emission to two pions which is well constrained experimentally. This matrix element, as will be shown shortly ahead, can be used to match gWEFT to a chiral EFT involving pions, $\chi$ EFT.

Specifically, since the quarkonium field $\phi$ is a scalar under chiral symmetry, the interaction operators with Goldstone bosons can be easily constructed making use as building blocks the unitary matrix $U(x)$, that parametrizes the Goldstone boson fields, and $\chi$ that involves the light-quark masses

$$
U=e^{i \Phi / F}, \quad \Phi=\left(\begin{array}{cc}
\pi^{0} & \sqrt{2} \pi^{+} \\
\sqrt{2} \pi^{-} & -\pi^{0}
\end{array}\right), \quad \chi=2 B \hat{m}_{q} \mathbf{1}
$$

where, at leading order, $F=F_{\pi}=92.419 \mathrm{MeV}$, and $\hat{m}_{q}=\left(m_{u}+m_{d}\right) / 2$. Working in the isospin limit, $m_{u}=m_{d}$, the pion mass is $m_{\pi}^{2}=2 B \hat{m} \approx(135 \mathrm{MeV})^{2}$. To leading order, the Lagrangian density of $\chi$ EFT can be written as [1]

$$
\begin{aligned}
& \mathcal{L}_{\chi \mathrm{EFT}}^{\phi}=\phi^{\dagger}\left(i \partial_{0}+\frac{\boldsymbol{\nabla}^{2}}{2 m_{\phi}}\right) \phi, \quad \mathcal{L}_{\chi \mathrm{EFT}}^{\pi}=\frac{F^{2}}{4}\left[\operatorname{Tr}\left(\partial_{\mu} U \partial^{\mu} U^{\dagger}\right)+\operatorname{Tr}\left(\chi^{\dagger} U+\chi U^{\dagger}\right)\right], \\
& \mathcal{L}_{\chi \mathrm{EFT}}^{\phi-\pi}=\phi^{\dagger} \phi \frac{F^{2}}{4}\left[c_{d 0} \operatorname{Tr}\left(\partial_{0} U \partial_{0} U^{\dagger}\right)+c_{d i} \operatorname{Tr}\left(\partial_{i} U \partial^{i} U^{\dagger}\right)+c_{m} \operatorname{Tr}\left(\chi^{\dagger} U+\chi U^{\dagger}\right)\right] .
\end{aligned}
$$

The are also $\phi$-contact interactions, but they do not play any role in the long-distance properties of quarkonium-quaktonium interaction in lowest order; they do play a role in the renormalization of ultraviolet divergences coming from chiral loops. The $c_{d 0}, c_{d i}$ and $c_{m}$ are matching coefficients that can be obtained from the matrix element [23]

$$
g^{2}\left\langle\pi^{+}\left(p_{1}\right) \pi^{-}\left(p_{2}\right)\left|\boldsymbol{E}_{a}^{2}\right| 0\right\rangle=\frac{8 \pi^{2}}{b}\left(\kappa_{1} p_{1}^{0} p_{2}^{0}-\kappa_{2} p_{1}^{i} p_{2}^{i}+3 m_{\pi}^{2}\right)
$$

where $\kappa_{1}=2-9 \kappa / 2, \kappa_{2}=2+3 \kappa / 2$, and $b=11 N_{c} / 3-2 N_{f} / 3$, and $\kappa$ is a parameter that can be obtained from pionic transitions of quarkonium states.

The matching of gWEFT to $\chi$ EFT is done by equating the amplitudes for two-pion production calculated in gWEFT and $\chi$ EFT, graphs (B) in Fig. 1:

$$
\frac{4 \pi^{2} \beta}{b}\left(\kappa_{1} p_{1}^{0} p_{2}^{0}-\kappa_{2} p_{1}^{i} p_{2}^{i}+3 m_{\pi}^{2}\right)=-c_{d 0} p_{1}^{0} p_{2}^{0}+c_{d i} p_{1}^{i} p_{2}^{i}-c_{m} m_{\pi}^{2},
$$

which yields

$$
c_{d 0}=-\frac{4 \pi^{2} \beta}{b} \kappa_{1}, \quad c_{d i}=-\frac{4 \pi^{2} \beta}{b} \kappa_{2}, \quad c_{m}=-\frac{12 \pi^{2} \beta}{b} .
$$

The chiral EFT just obtained can be employed to predict the leading chiral logarithm contribution to the quarkonium mass. Such a dependence is useful in extrapolations to the physical limit lattice results obtained with higher pion masses [24]. The calculation is straightforward; using dimensional regularization, the result is

$$
\left.\delta m_{\phi}\right|_{\text {chiral } \log }=-\frac{3}{8} \frac{\beta}{b} m_{\pi}^{4} \log \frac{m_{\pi}^{2}}{v^{2}},
$$

where $v$ is a renormalization scale. A similar calculation was performed in Ref. [24], but the result obtained in that reference disagrees with Eq. (15): our results is smaller by a factor 16. Possible sources for the disagreement are discussed in Ref. [1]. 


\section{From $\chi$ EFT to WEFT: van der Waals potential}

If one is interested in a quarkonium-quarkonium process with momentum transfers $k_{\phi \phi} \sim m_{\pi}$, one can integrate out the pions. This is because the energy scale of the two-quarkonia process mediated by pions, which is given by the relative kinetic energy, is much smaller than the pion mass:

$$
\frac{k_{\phi \phi}^{2}}{m_{\phi}^{2}} \sim \frac{m_{\pi}^{2}}{m_{\phi}^{2}} \ll m_{\pi} .
$$

Integrating out the pion, one obtains a new EFT at the scale of the kinetic energy operator of the $\phi$ field, that we name van der Waals EFT (WEFT); the matching "coefficient" in WEFT is the van der Waals potential— the matching is illustrated by the graphs (C) in Fig. (1).

At leading order, the Lagrangian of WEFT is given by

$$
L_{\mathrm{WEFT}}=\int d \boldsymbol{R} \phi^{\dagger}(t, \boldsymbol{R})\left(i \partial_{0}+\frac{\boldsymbol{\nabla}^{\mathbf{2}}}{2 m_{\phi}}\right) \phi(t, \boldsymbol{R})-\frac{1}{2} \int d \boldsymbol{R}_{1} d \boldsymbol{R}_{2} \phi^{\dagger} \phi\left(t, \boldsymbol{R}_{\mathbf{1}}\right) W\left(\boldsymbol{R}_{\mathbf{1}}, \boldsymbol{R}_{2}\right) \phi^{\dagger} \phi\left(t, \boldsymbol{R}_{\mathbf{2}}\right) .
$$

where chiral corrections to the quarkonium mass have been reabsorbed in a field redefinition. As said above, the potential $W\left(\boldsymbol{R}_{\mathbf{1}}, \boldsymbol{R}_{\mathbf{2}}\right)$ is obtained by matching $\chi$ EFT to WEFT. In practice, the potential is first calculated in momentum space; the short range part is dominated by contact terms, which include renormalization counterterms; the long range part depends only on the $\boldsymbol{k}^{2}=\left(\boldsymbol{p}-\boldsymbol{p}^{\prime}\right)^{2}$, where $\boldsymbol{p}$ and $\boldsymbol{p}^{\prime}$ are the initial and final c.m. momenta of the bottomonia. Since we are interested only in the long-range part, to implement the Fourier transform of the momentum-space potential it is convenient to use a dispersive representation $[25,26]$ that allows us to separate the long-range contributions. After a somewhat lengthly, but straightforward calculation, one can write an exact expression for the long-range potential in coordinate space-for details see Ref. [1]:

$$
\begin{aligned}
W(r)= & -\frac{3 \pi \beta^{2} m_{\pi}^{2}}{8 b^{2} r^{5}}\left\{\left[4\left(\kappa_{2}+3\right)^{2}\left(m_{\pi} r\right)^{3}+\left(3 \kappa_{1}^{2}+43 \kappa_{2}^{2}+14 \kappa_{1} \kappa_{2}\right) m_{\pi} r\right] K_{1}\left(2 m_{\pi} r\right)\right. \\
& \left.+2\left[2\left(\kappa_{2}+3\right)\left(\kappa_{1}+5 \kappa_{2}\right)\left(m_{\pi} r\right)^{2}+\left(3 \kappa_{1}^{2}+43 \kappa_{2}^{2}+14 \kappa_{1} \kappa_{2}\right)\right] K_{2}\left(2 m_{\pi} r\right)\right\}
\end{aligned}
$$

where $r=\left|\boldsymbol{R}_{1}-\boldsymbol{R}_{2}\right|$, and $K_{1}(x)$ and $K_{2}(x)$ are the modified Bessel functions of the second kind. For large $r$, the potential is

$$
W(r) \stackrel{r \gg 2 m_{\pi}}{\longrightarrow}-\frac{3\left(3+\kappa_{2}\right)^{2} \pi^{3 / 2} \beta^{2}}{4 b^{2}} \frac{m_{\pi}^{9 / 2}}{r^{5 / 2}} e^{-2 m_{\pi} r} .
$$

Numerical results are discussed in the next section.

\section{Numerical results}

We start presenting explicit results for the chromopolarizabilty $\beta$, derived in Section 2 . At leading order, the bound-state wave functions are simply the hydrogen-atom wave functions with the finestructure constant $\alpha_{\text {e.m. }}$. replaced by $C_{F} \alpha_{s}$. The continuum Coulomb wave functions can be found in Ref. [27]. At leading order, the mass of the heavy quarkonium is given by $m_{\phi}=2 m-m\left(C_{F} \alpha_{s}\right)^{2} / 4$. Taking the mass of $\phi$ as the spin average of the $\eta_{c}$ [28] and $\Upsilon(1 \mathrm{~S})$ [29] masses, i.e. $m_{\phi}=9.4454 \mathrm{GeV}$, and using the conventional value $m=5 \mathrm{GeV}$ for the bottom mass, we get $\alpha_{\mathrm{s}} \approx 0.5$. The natural scale of $\alpha_{\mathrm{s}}$ for the binding energy is of the order of the inverse Bohr radius; to assess the impact of uncertainties in the value of $\alpha_{s}$, we use in the calculation of $\beta$ as a central value $\alpha_{s}(1.5 \mathrm{GeV}) \approx 0.35$, 
and $\alpha_{s}(2 \mathrm{GeV}) \approx 0.3$ and $\alpha_{s}(1 \mathrm{GeV}) \approx 0.5$ as lowest and largest values. Using $m=5 \mathrm{GeV}$ and $N_{c}=3$, these yield for $\beta[1]$

$$
\beta=0.50_{-0.38}^{+0.42} \mathrm{GeV}^{-3}
$$

Of course, there are further uncertainties associated with the bottom mass and higher-order corrections. One can compare our results with a related calculation [30] based on the transition $\Upsilon(2 S) \rightarrow \Upsilon(1 S) \pi \pi$ that finds $\beta_{\Upsilon-\Upsilon^{\prime}}=0.66 \mathrm{GeV}^{-3}$, computed using the QCD trace anomaly and fitting experimental data on the decay rates. Although $\beta$ and $\beta_{\Upsilon-\Upsilon^{\prime}}$ do not correspond to the same quantity, the numbers in Eq. (20) are similar to this determination—actually, one could obtain $\beta=0.66 \mathrm{GeV}^{-3}$ using $\alpha_{s} \simeq 0.326$.

The effect of using continuum Coulomb wave functions instead of noninteracting plane-wave as intermediate states in the evaluation of $\beta$ can be seen in Fig. 2. From the figure, one can see that the effect of using continuum Coulomb wave functions instead of plane waves is to increase the polarizability; the increase is by $\sim 5 \%$.

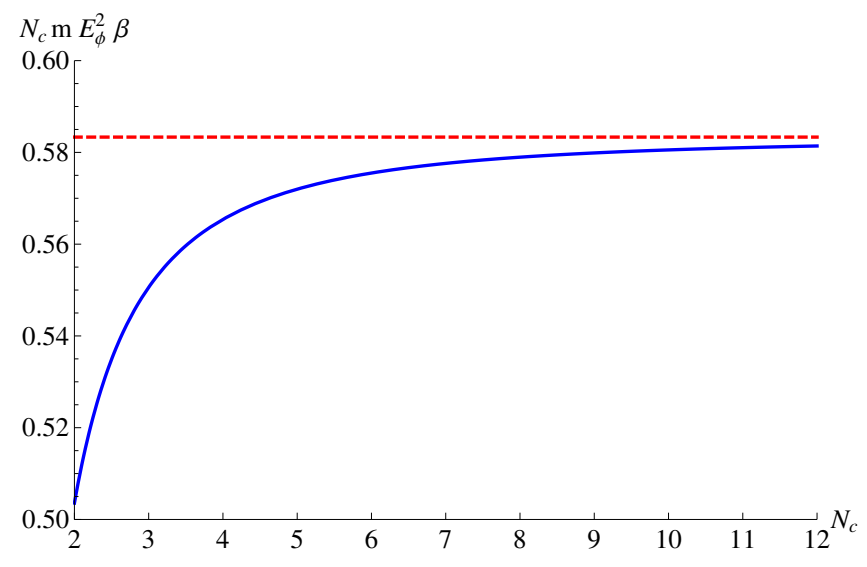

Figure 2. The chromopolarizability as a function of $N_{c}$. Graph from Ref. [1]

Next, we consider the vdW potential. Initially, we remark that an earlier result derived in Ref. [31] using a similar method to ours based on the dipole-dipole interaction and the trace anomaly is contained in our exact expression for the long-range part of the potential, Eq. (19), when one takes $\kappa_{2}=2$ in that equation and also neglect contributions proportional to $m_{\pi}^{2}$ in the trace anomaly. We note that while the functional dependence on $r$ and $m_{\pi}$ of the potential is not modified when neglecting $m_{\pi}^{2}$ in the trace anomaly, the strength of the long-range van der Waals is modified: the van der Waals potential in Ref. [31] is a factor 16/25 weaker than ours.

To conclude, in Fig. 3 shows a comparison between Eqs. (18) and (19) for $r \leq 2 \mathrm{fm}$. While the longest-range part of the vdW potential, as indicated in Eq. (19), is valid for $r \gg 1 / 2 m_{\pi}$, the full expression in Eq. (18) is also valid for $r \simeq 1 / 2 m_{\pi} \sim 0.7 \mathrm{fm}$ and it may be as large a $-1 \mathrm{MeV}$. Clearly, the vdW potential that we have obtained is much weaker than the analogue from the two-pion exchange in the nucleon-nucleon interaction. On the other hand, since the bottomonium mass is much larger that the nucleon mass, the existence of two-bottomonium bound states is not discarded, but admittedly the perspectives are not very promising. For a detailed study, the short-distance parts need to be considered. 


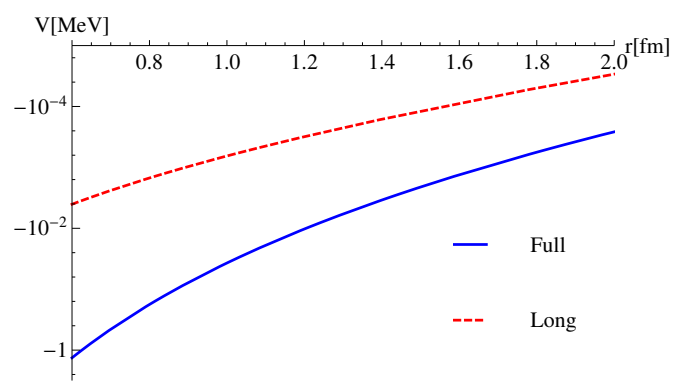

Figure 3. Comparison of expressions in Eqs. (18) (blue line) and (18) (blue line) for the vdW potential. Here we use $\beta=0.92 \mathrm{GeV}^{-3}$, and the other parameters are $\kappa=0.186, m_{\pi}=135 \mathrm{MeV}$ and $b$ is computed with $N_{f}=N_{c}=3$. Graph from Ref. [1]

\section{Conclusions and perspectives}

In this communication we have presented selected results from a recent study [1] of long-range properties of bottomonium. The study was conducted within an EFT framework. We focused on the $1 S$ states, which are the lowest lying in the bottomonium spectrum. The analysis relied on an assumed hierarchy of scales, namely $m v \gg m v^{2} \gg \Lambda_{\mathrm{QCD}}$. Of course, such an assumption can be questioned, but once assumed, our results follow rigorously. Our final aim in the work was the derivation of the long-range part of the effective interaction between two bottomonia.

At the ultrasoft energy scale, $m v^{2}$, the $1 S$ bottomonium states are solutions of the Schrödinger equation defined from the EFT known as weakly coupled pNRQCD. Because of the large mass of the bottom quark, spin-dependent interactions are suppressed, we considered the $1 S$ states $\eta_{b}$ (spin singlet) and $\Upsilon(1 S)$ (spin triplet) as being degenerate and describe them by a $0^{-+}$field $\phi$. Integrating out the ultrasoft scale, one obtain an EFT, named gWEFT, whose dynamical degrees of freedom are the $\phi$ and the gluon fields. The matching of pNRQCD to gWEFT leads to a coupling between $\phi$ and chromoelectric fields given by the chromopolarizability $\beta$, which can be computed in perturbation theory. A key ingredient in the calculation of $\beta$, Eq. (8), is the use of continuum Coulomb coloroctet states, instead of plane waves as usually done in the literature; we found that the effect of using the Coulomb wave functions is to increase $\beta$ by $5 \%$ for $N_{c}=3$. The expression found agrees with previous findings in the literature.

At the level of gWEFT, the gluon dynamics is nonperturbative. Pursuing the aim of deriving the $\phi-\phi$ interaction, we have used the QCD trace anomaly to match gWEFT to a chiral EFT, $\chi$ EFT, in which the $\phi$ 's and pions are the degrees of freedom. For the derivation of the long-range van der Waals potential generated by the two-pion exchange between two $\phi$ 's, whose dynamics is set at a lower scale than the pion dynamics, namely the relative kinetic energy of the $\phi-\phi$ system, we integrated out the pion from $\chi$ EFT and obtained a new EFT, named WEFT, where the potential is the corresponding matching coefficient. The $\phi-\phi$ long-range potential we obtained shows a significant dependence on the value of the polarizability $\beta$. The long-range parametric dependence on the distance $r$ of the potential resembles that of the two-pion exchange potentials of the nucleon-nucleon interaction, its actual strength reflects the uncertainty on $\beta$. The possible existence of a $\phi-\phi$ bound state will also depend crucially on the $\phi-\phi$ short-range interaction, which needs to be addressed in a more complete work. 


\section{Acknowledgments}

Special thanks to Nora Brambilla, Jaume Tarrús-Castellà and Antonio Vairo for the collaborative work. Work partially financed by Conselho Nacional de Desenvolvimento Científico e Tecnológico - CNPq, Grant Nos. 305894/2009-9 and Fundação de Amparo à Pesquisa do Estado de São Paulo - FAPESP, Grant No. 2013/01907-0. A grant from the bilateral agreement between Bayerische Hochschulzentrum für Lateinamerika (BAYLAT) of the Bayerischen Staatsministeriums für Bildung und Kultus, Wissenschaft und Kunst (StMBW) and Fundação de Amparo à Pesquisa do Estado de São Paulo (FAPESP), Contracts No. 914-20.1.3 (BAYLAT) and No. 2013/50841-1 (FAPESP) is acknowledged.

\section{References}

[1] N. Brambilla, G. Krein, J. Tarrús Castellà and A. Vairo, Phys. Rev. D 93, 054002 (2016).

[2] N. Brambilla et al., Eur. Phys. J. C 74, 2981 (2014).

[3] R. A. Briceno et al., Chin. Phys. C 40, 042001 (2016).

[4] S. Dubynskiy and M. B. Voloshin, Phys. Lett. B 666, 344 (2008).

[5] S. J. Brodsky, I. A. Schmidt, and G. F. de Teramond, Phys. Rev. Lett. 64, 1011 (1990).

[6] S. R. Beane, et al. Phys. Rev. D 91, 114503 (2015).

[7] G. F. de Teramond, R. Espinoza, and M. Ortega-Rodriguez, Phys. Rev. D 58, 034012 (1998).

[8] G. Krein, A.W. Thomas, and K. Tsushima, Phys. Lett. B 697, 136 (2011).

[9] K. Tsushima, D. H. Lu, G. Krein, and A.W. Thomas, Phys. Rev. C 83, 065208 (2011).

[10] N. Brambilla, A. Pineda, J. Soto, and A. Vairo, Rev. Mod. Phys. 77, 1423 (2005).

[11] S. Weinberg, Physica A 96, 327 (1979).

[12] J. Gasser and H. Leutwyler, Ann. Phys. 158, 142 (1984).

[13] W. E. Caswell and G. P. Lepage, Phys. Lett. B 167, 437 (1986).

[14] G. T. Bodwin, E. Braaten, and G. P. Lepage, Phys. Rev. D 51, 1125 (1995); 55, 5853(E) (1997).

[15] A. Pineda and J. Soto, Nucl. Phys. B, Proc. Suppl. 64, 428 (1998).

[16] N. Brambilla, A. Pineda, J. Soto, and A. Vairo, Nucl. Phys. B566, 275 (2000).

[17] A. Pineda, Prog. Part. Nucl. Phys. 67, 735 (2012).

[18] A. Vairo, in Quark Confinement and the Hadron Spectrum IV, edited by W. Lucha and K. M.

Maung (World Scientific, Singapore, 2002), p. 221.

[19] M. E. Peskin, Nucl. Phys. B156, 365 (1979).

[20] G. Bhanot and M. E. Peskin, Nucl. Phys. B156, 391 (1979).

[21] M. B. Voloshin, Nucl. Phys. B154, 365 (1979).

[22] H. Leutwyler, Phys. Lett. B 98, 447 (1981).

[23] M. B. Voloshin, Prog. Part. Nucl. Phys. 61, 455 (2008).

[24] B. Grinstein and I. Z. Rothstein, Phys. Lett. B 385, 265 (1996).

[25] N. Kaiser, R. Brockmann, and W.Weise, Nucl. Phys. A 625, 758 (1997).

[26] E. Epelbaum, W. Gloeckle, and U.-G. Meißner, Eur. Phys. J. A 19, 125 (2004).

[27] M. Abramovitz and I. A. Stegun, Handbook of Mathematical Functions with Formulas, Graphs and Mathematical Tables (Dover, New York, 1964).

[28] U. Tamponi et al. (Belle Collaboration), Phys. Rev. Lett. 115, 142001 (2015).

[29] K. A. Olive et al. (Particle Data Group), Chin. Phys. C 38, 090001 (2014).

[30] M. B. Voloshin, Mod. Phys. Lett. A 19, 665 (2004).

[31] H. Fujii and D. Kharzeev, Phys. Rev. D 60, 114039 (1999). 